\title{
Syndrome de Beckwith-Wiedemann : évolution favorable des troubles fonctionnels après glossectomie
}

\author{
Zineb Jaja ${ }^{1}$, Mohamed Boulaich ${ }^{2}$, Ali El Ayoubi ${ }^{2, \star}$, Fahd El Ayoubi ${ }^{2}$, Salma Kettani ${ }^{1}$, \\ Laïla Essakalli², Mohamed Kzadri ${ }^{2}$
}

1 Service de Réanimation pédiatrique, Hôpital des enfants, Rabat, Maroc

2 Service d'ORL et Chirurgie maxillo-faciale, Rabat, Maroc

(Reçu le 11 novembre 2010, accepté le $1^{\text {er }}$ décembre 2010 )

\author{
Mots clés : \\ macroglossie / \\ syndrome \\ de Beckwith-Wiedemann / \\ glossectomie \\ partielle \\ Key words: \\ macroglossia / \\ Beckwith-Wiedemann \\ syndrome / \\ partial glossectomy
}

\begin{abstract}
Résumé - La macroglossie est un symptôme qui peut être associé à différentes affections; elle est souvent responsable de troubles fonctionnels (déglutition, respiration, phonation), d'intensité variable. Nous rapportons l'observation d'un enfant atteint d'un syndrome de Beckwith-Wiedemann et porteur d'une volumineuse macroglossie. La résection partielle de la langue a entrainé une amélioration spectaculaire des troubles fonctionnels.
\end{abstract}

\begin{abstract}
Surgical reduction of congenital macroglossia in the Beckwith-Wiedemann syndrome with dramatic results. Macroglossia is a symptom associated with various pathologies and it is often responsible for disorders of digestive, respiratory and phonatory functions.

We report a case of a child with macroglossia in the Beckwith-Wiedemann syndrome and whom a partial resection of tongue was realized with dramatic results.
\end{abstract}

Une macroglossie, congénitale ou acquise, peut être observée dans diverses affections; les formes congénitales dans la trisomie 21, l'hypothyroïdie congénitale... et le syndrome de Beckwith-Wiedemann. Elle est souvent responsable de troubles fonctionnels (déglutition, respiration, phonation) d'intensité variable [1].

Nous rapportons l'observation d'un enfant atteint d'un syndrome de Beckwith-Wiedemann avec une volumineuse macroglossie. Après une glossectomie partielle, on a observé une évolution rapidement favorable des troubles fonctionnels.

\section{Observation}

Il s'agit d'un enfant âgé de 2 ans, issu d'un mariage consanguin au $3^{\text {ème }}$ degré. La grossesse a été bien suivie et l'accouchement effectué par césarienne car le terme était considéré comme dépassé. A la naissance, il a été admis immédiatement en réanimation pédiatrique pour détresse respiratoire : il a été intubé et ventilé puis, à la fin de la période d'intubation, une canule de Krishaberg a été mise en place.

\footnotetext{
^Correspondance : alineijou@hotmail.com
}

L'examen clinique a révélé une macroglossie importante avec protrusion permanente de la langue, une macrosomie, une légère dysmorphie faciale (Fig. 1). La glycémie néonatale était normale ainsi que le bilan thyroïdien et l'échographie abdominale. Le tableau clinique était évocateur d'un syndrome de Beckwith-Wiedemann.

En raison du retentissement fonctionnel de la macroglossie, une glossectomie partielle a été réalisée (Figs. 2 et 3). Les suites postopératoires ont été simples. L'amélioration des fonctions respiratoires a permis de décanuler l'enfant 5 mois après l'intervention (Fig. 4).

\section{Discussion}

La macroglossie représente un élément constitutif du syndrome de Beckwith-Wiedemann qui a été décrit en 1963-1964, indépendemment par $\mathrm{J}$ Bruce Beckwith, pathologiste pédiatrique américain, et par Hans-Rudolf Wiedemann, pédiatre allemand, qui avait proposé le terme de syndrome EMG pour exomphalos-macroglossia-gigantism.

Aux USA, la prévalence du syndrome de BeckwithWiedemann est de 7,3/100000 (300 nouveaux cas chaque 


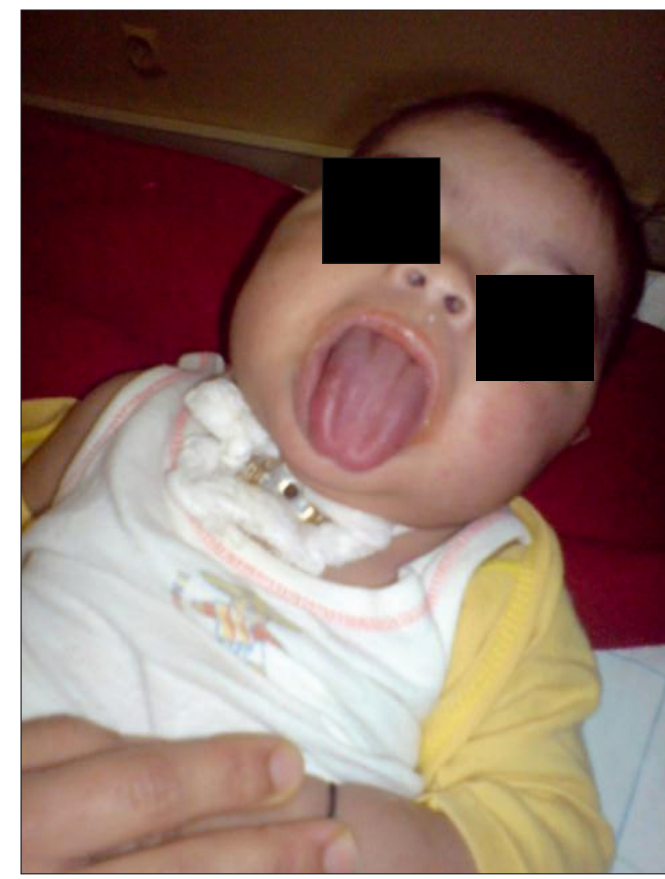

Fig. 1. Aspect de la macroglossie à l'âge d'1 an. Fig. 1. Macroglossia at the age of 1 year.

année), et le risque 3 à 4 fois plus élevé après fécondation in vitro. Le mode de transmission est autosomique dominant, mais $85 \%$ des cas correspondent à la forme sporadique. Dans 7 cas sur 10, on retrouve une mutation génétique (région 11p15) ou épigénétique.

Ce syndrome comporte cinq anomalies principales - une macroglossie, une macrosomie (parfois associée à une viscéromégalie), des anomalies de la ligne médiane abdominale (omphalocèle, hernie ombilicale, diatasis recti), des anomalies du pavillon de l'oreille (puits et plis cutanés) et une hypoglycémie néonatale - et une trentaine d'anomalies secondaires - naevus flammeus facial, occipital proéminent, anomalies génito-urinaires (néphromégalie...) anomalies cardiaques, anomalies musculo-squelettiques, surdité, hydramnios... Dans le cas rapporté, la macrosomie pré et néo-natale a probablement fait porter à tort le diagnostic de terme dépassé.

Comme il existe de grandes variations dans les manifestations du syndrome de Beckwith-Wiedemann, plusieurs définitions du syndrome ont été proposées. Avec celle de Elliot et al. par exemple, on doit retrouver 3 anomalies majeures (une macroglossie, une anomalie de la paroi abdominale médiane, une macrosomie pré ou néo-natale) ou deux anomalies majeures et trois mineures (puits cutanés dans le pavillon de l'oreille, naevus flammeus, hypoglycémie néo-natale, néphromégalie ou hémihypertrophie corporelle) [2]. Selon Williams et al. [2], la macroglossie constitue un des critères majeurs pour le diagnostic avec l'hémihypertrophie corporelle et les anomalies de la paroi abdominale; les critères mineurs comprennent une dysgénésie rénale ou une néphromégalie, une

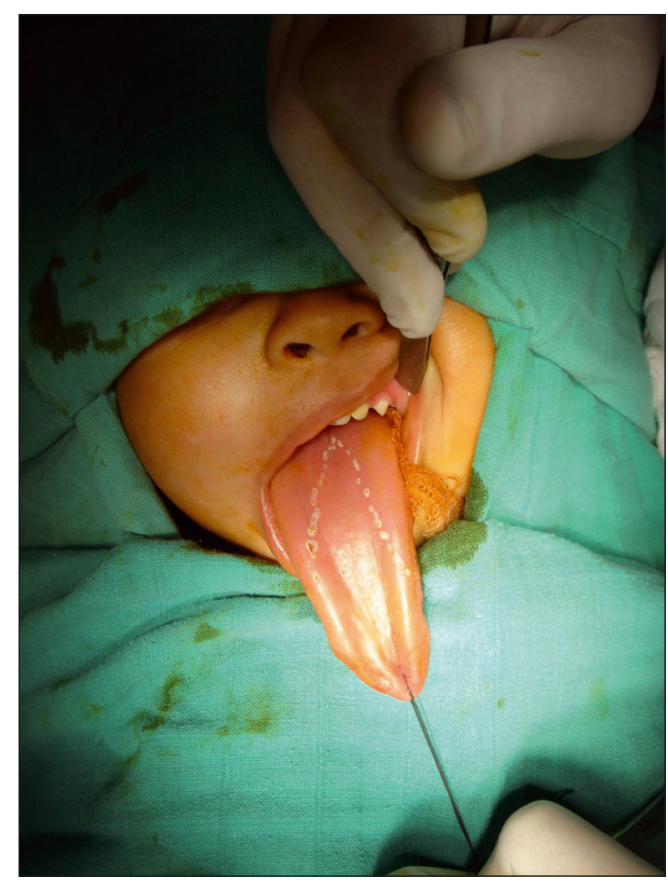

Fig. 2. Tracé de la glossectomie.

Fig. 2. Plan of glossectomy.

cytomégalie surrénalienne, une anomalie chromosomique et un hydramnios... La fréquence des principales manifestations est présentée dans le Tab. I.

Les enfants atteints d'un syndrome de Beckwith-Wiedemann développent plus de tumeurs embryonnaires (néphroblastome, hépatoblastome...) principalement dans la petite enfance : $80 \%$ des tumeurs apparaissent avant l'âge de 4 ans, les autres avant l'âge de 10 ans. Après cet âge, l'incidence des cancers est identique à celle de la population générale. Un peu plus de $10 \%$ (ou 7,5 \% cf. Tab. I) des enfants atteints d'un syndrome de Beckwith-Wiedemann développent un cancer et, dans 50 à $70 \%$ des cas, il s'agit d'un néphroblastome (tumeur de Wilms). La plupart des auteurs conseillent donc de réaliser une échographie abdominale tous les 3 à 6 mois et un dosage de l' $\alpha$-foeto-proteine toutes les 6 semaines.

Dans notre domaine, la prise en charge consiste en une réduction de la macroglossie [5], des conseils hygiénodiététiques et la planification d'une surveillance périodique clinique et échographique tous les 3 à 6 mois pour dépister le développement d'une éventuelle tumeur.

Comme dans le cas rapporté, la gêne respiratoire (obstruction des voies aériennes) représente la principale indication pour la glossectomie [5]. La résection partielle constitue la technique de base pour le traitement des macroglossies [5] : elle a pour but d'obtenir une diminution suffisante de la taille de la langue tout en préservant ses fonctions. Pour cet enfant, on a utilisé la technique de la résection cunéiforme de la langue mobile. La radiofréquence, qui permet de réduire le volume de la base de la langue, est une option thérapeutique 


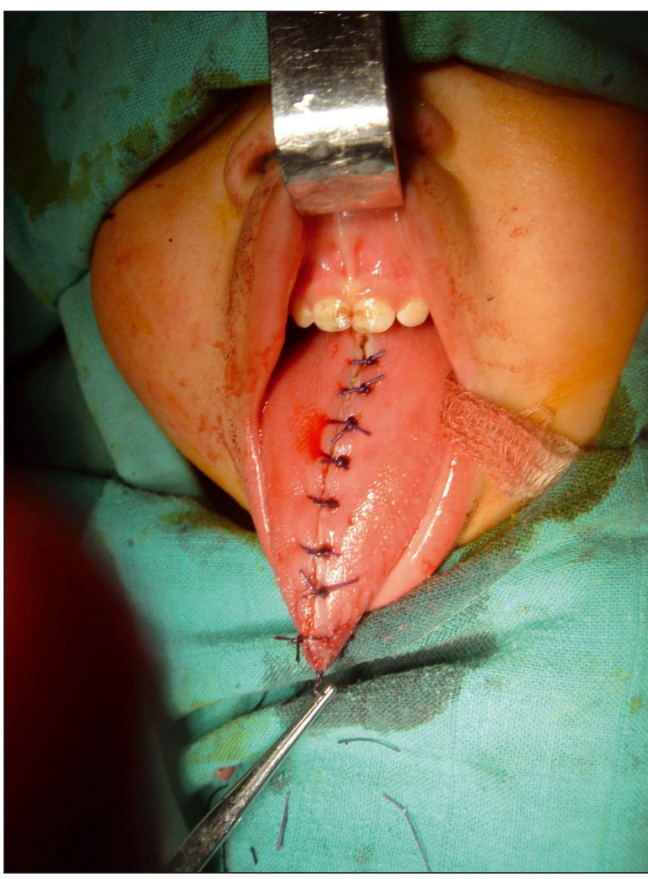

Fig. 3. Aspect de la langue à la fin de l'intervention chirurgicale. Fig. 3. Apparence of the tongue after surgical intervention.

Tableau I. Frequence des principales manifestations observées dans le syndrome de Beckwith-Wiedemann [3].

Table I. Frequency of principal manifestations in Beckwith-Wiedemann syndrome [3].

\begin{tabular}{|l|c|}
\hline \multicolumn{1}{|c|}{ Manifestations majeures } & $\%$ \\
\hline Macroglossie & $95 \%$ \\
\hline Macrosomie ou excès de croissance & $80 \%$ \\
\hline Anomalies de la ligne médiane abdominale & $65 \%$ \\
\hline Organomégalie & $50 \%$ \\
\hline
\end{tabular}

\begin{tabular}{|l|c|}
\hline \multicolumn{1}{|c|}{ Manifestations cliniques mineures } & $\%$ \\
\hline Hypoglycémie néonatale & $40 \%$ \\
\hline Anomalies rénales & \\
\hline $\begin{array}{l}\text { Anomalies du pavillon de l'oreille } \\
\text { (puits et plis cutanés) }\end{array}$ & $30 \%$ \\
\hline Naevus flammeus facial & $30 \%$ \\
\hline Hémihyperplasie & 30 à $35 \%$ \\
\hline Polyhydroamnios & $30,5 \%$ \\
\hline$\ldots$ & \\
\hline Tumeurs embryonnaires & $7,5 \%$ \\
\hline
\end{tabular}

nouvelle en cours d'évaluation chez les enfants ayant une macroglossie. Les études réalisées par Powell et al. ont montré que cette technique réduit la taille de la langue permettant l'amélioration des troubles fonctionnels [7].

\section{Conclusion}

Le syndrome de Beckwith-Wiedemann se caractérise par une croissance excessive, en particulier de la langue. La

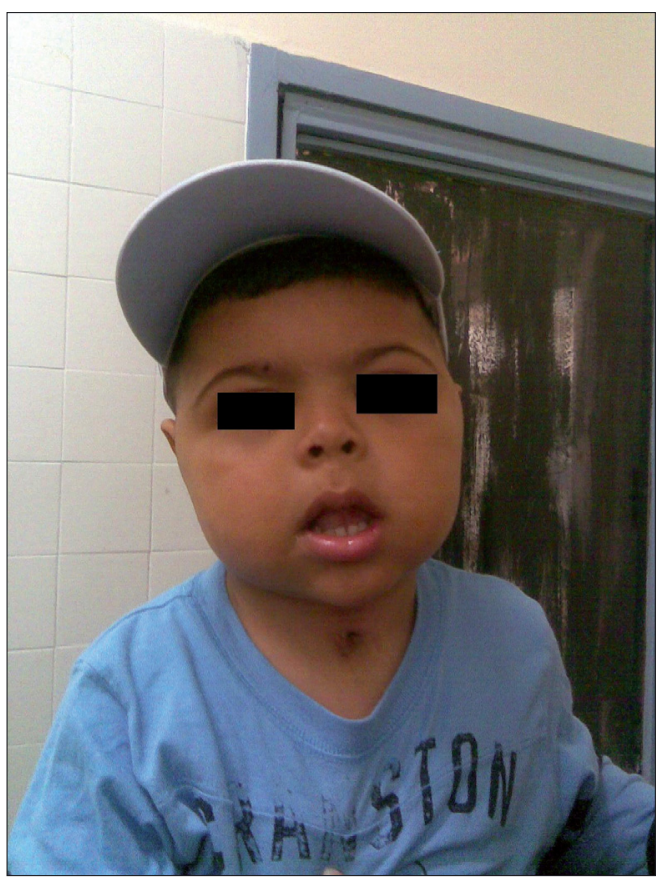

Fig. 4. Enfant 5 mois après la glossectomie.

Fig. 4. Child 5 months after surgery.

macroglossie peut être responsable de troubles fonctionnels touchant la déglutition, la respiration et la phonation. L'importance des troubles conduit parfois à réaliser une réduction chirurgicale du volume de la langue.

Conflits d'intérêt : aucun

\section{Références}

1. Unachak K, Dejkhamron P. Primary congenital hypothyroidism: clinical characteristics and etiological study. J Med Assoc Thai 2004;87:612-7.

2. Elliott M, Bayly R, Cole T, Temple TK, Maher ER. Clinical features and natural history of Beckwith-Wiedemann syndrome : presentation of 74 new cases. Clin Genet 1994;46:168-74.

3. Gicquel C, Rossignol S, Le Bouc Y. Beckwith-Wiedemann syndrome. Orphanet encyclopedia. March 2005. http://www.orpha.net/data/patho/GB/uk-bws05.pdf

4. Ndiaye 0 , Diouf $S$, Fall AL, Sylla A, Guèye M, Ouattara A, Sall MG, Kuakuvi N. Beckwith-Wiedemann syndrom: a case report in Dakar. Dakar Med 2006;51:101-3.

5. Myer CM, Hotaling AJ, Reilly JS. The diagnosis and treatement of macroglossia in children. Ear Nose Throat J 1986;65:444-8.

6. Sally R, Shott MD. Operatives techniques in otolyryngology. Head Neck Surg 2001;12:210-3.

7. Powell NB, Riley RW, Guilleminault C. Radiofrequency tongue base reduction in sleep-disordered breathing : a pilot study. Otolaryngol Head Neck Surg 1999;120:656-64. 